\title{
Participation Politique Des Femmes Au Niger: Analyse De La Contribution Du Genre Féminin Dans Le Développement Politique
}

\author{
Zakari Aboubacar \\ Maître-assistant, Sociologue, Département de Sociologie, Faculté des Lettres \\ et Sciences Humaines, Université de Zinder, Niger
}

doi: 10.19044/esj.2017.v13n16p99 URL:http://dx.doi.org/10.19044/esj.2017.v13n16p99

\begin{abstract}
This research focuses on the political participation of women in Niger. In addition to the documentation on the subject, we conducted interviews with women and political party leaders. Thus, the quest for equality is more than ever a concern of Nigerian women. In this article we asked the following research question: how can we explain the contribution of the feminine gender to political development in Niger? The general objective pursued through this article is to analyze the contribution of women in political development in Niger. To answer the research question and check the general objective the qualitative method through maintenance guides was used. After analyzing the data, the results show that politics is still an exclusively male domain or that there are many institutionalized discriminations, the dominance of political space by men being the rule, although it did not exclude a certain presence women. The results also show that the question of the presence of women in public life in general and political space in particular is a global challenge to harmonize the political space of Niger.
\end{abstract}

Keywords: Political participation, gender contribution, Political development, Niger, democracy

\section{Résumé}

La présente recherche traite de la participation politique des femmes au Niger. En plus de la documentation sur le sujet nous avons réalisé des entretiens avec des femmes et des responsables des partis politiques. Ainsi, la quête d'égalité est aujourd'hui plus que jamais une préoccupation des femmes nigériennes. Dans le présent article nous avons posé la question de recherche suivante : comment peut-on expliquer la contribution du genre féminin dans le développement politique au Niger ? L’objectif général 
poursuivi à travers cet article est d'analyser la contribution des femmes dans le développement politique au Niger. Pour répondre à la question de recherche et vérifier l'objectif général la méthode qualitative à travers des guides d'entretien a été utilisé. Après l'analyse des données, les résultats montrent que Le politique reste encore un domaine exclusivement masculin ou existaient de nombreuses discriminations institutionnalisées, la domination de l'espace politique par les hommes étant la règle, même si elle n'excluait pas une certaine présence des femmes. Les résultats démontrent aussi que la question de la présence des femmes dans la vie publique de manière générale et l'espace politique en particulier est un défi global pour harmoniser l'espace politique nigérien.

Mot-clés: Participation politique, contribution du genre, développement politique, Niger, démocratie

\section{Introduction}

Au Niger, la question du genre est une préoccupation permanente des différents régimes et gouvernements qui se sont succédé. Elle est aussi un combat sans cesse mené par la société civile, les Partenaires Techniques et Financiers (PTF) dans la promotion, la préservation et la sauvegarde des droits de la femme. Dans le même cadre, à l'instar des autres pays africains, le Niger a ratifié plusieurs textes internationaux. Au niveau national, différents textes et politiques ont été élaborés pour soutenir la promotion du genre.

Ainsi à travers cette recherche intitulée: "Participation politique des femmes au Niger : Analyse de la contribution du genre féminin dans le développement politique ", nous posons la question de recherche qui suit : comment peut-on expliquer la contribution du genre féminin dans le développement politique au Niger ? Cette question nous permettra de ressortir la contribution du genre féminin au développement politique du pays.

\section{Méthodologie d'analyse}

Pour rendre compte de façon analytique cette étude, en plus des textes et des écrits sur la problématique, nous avons réalisé des entretiens avec une grille d'entretien sous la forme semi structurée. Ces entretiens ont permis de faire le tour de la question car émanant des différents acteurs concernés par le système. En ce qui concerne la méthode d'analyse, un guide d'entretien a été administré. Concernant le modèle d'analyse, nous avons fondé notre travail sur le modèle wébérien, c'est-à-dire la méthode compréhensive. En effet, WEBER est considéré comme le défenseur de cette approche. Pour lui, un phénomène social se saisit dans la signification que 
les individus donnent à leurs actes en ce sens que l'homme est un être de conscience, qui agit en fonction de sa compréhension du monde et des intentions qu'il a. Analyser le social, c'est donc partir de ces actions et des intentions qui les constituent.

\section{Définition des concepts}

Participation politique: La participation politique désigne l'ensemble des activités d'ordre politique que peuvent avoir les individus au sein d'une société. L'implication du citoyen dans la vie politique institutionnalisée est couramment appelée la participation conventionnelle. Dans le cadre de cette recherche, il faut mettre l'accent autour de l'élection, et donc de l'acte de vote que s'ordonne cette participation conventionnelle, mais nous pouvons étendre celle-ci à tout ce qui concourt à produire le processus électoral (intérêt porté à la vie politique par l'écoute des émissions politiques, lecture de la presse, discussions et débats politiques, participation à des meetings, soutient à des partis, adhésion et militantisme).

Contribution du genre féminin: c'est le rôle joué par la femme dans ce processus du développement politique. De façon pratique, l'objectif général de la Politique Nationale Genre est de promouvoir un développement participatif et équitable des hommes et des femmes, en leur assurant un accès et un contrôle égal et équitable aux ressources et aux sphères de décision, dans le respect de leurs droits fondamentaux". "Le genre dépasse une simple démarche en termes de modèle de développement : il s'agit d'un phénomène qui pénètre le cœur de notre existence et de notre vécu quotidien. C'est pourquoi le genre veut être une pratique à observer dans la vie sociale, économique et politique." "Le genre est une question d'adhésion et d'engagement individuel et collectif. La mobilisation sociale de tous les acteurs et la mobilisation conséquente des ressources sont vitales dans le processus d'institutionnalisation du genre."

Développement politique: Par développement politique, il faut entendre le progrès enregistré en matière politique, au processus de la démocratie dans un pays donné. Ainsi, chaque politique des pouvoirs publics, pour parvenir au développement d'un pays, répond à une option économique prédéfinie, pour des objectifs précis, à partir des stratégies également bien définies. Ici, il est surtout question de l'apport des femmes dans la stratégie globale du développement d’un pays.

\section{Etat de connaissance du problème}

\section{Aperçu général sur la question}

La problématique de la participation politique des femmes est diversement appréciée par les chercheurs classiques et modernes. Selon la discipline, les aspects abordés ne sont forcément appréhendés avec la même 
méthodologie, et les contextes diffèrent dans la plupart des cas. C’est ainsi que Sabine Manigat (2013), aborde la question sur le gain de la participation politique des femmes.

Son article pose la question du rapport des femmes à la politique. L’expérience historique révèle que l’égalité ne découle pas naturellement du droit et le féminisme analyse donc les rapports de genre en fonction de la question du pouvoir. Cette contribution scientifique relève le cas de Haïti ; et à ce propos, trois questions sont débattues : Qu'est-ce qui conditionne la participation politique des femmes ? Des mesures spéciales pour promouvoir cette participation sont-elles légitimes ? Et surtout : les femmes font-elles la politique autrement ? à travers ces question l'auteur présente des résultats intéressants : L'exclusion des femmes de certains espaces et leur rôle social limité conditionnent toute participation. L'inclusion sociale des femmes constitue une bataille ardue. Celle-ci passe largement par l'éducation et par la lutte contre les violences qui inhibent et réduisent les femmes. L'intériorisation de la domination par les victimes elles-mêmes aggrave la domination subie. Pour contrer cette situation, Sabine (2013) stipule sue des mesures transitoires śavèrent nécessaires, comme le quota de femmes dans la vie nationale, récemment adopté par Haïti. Ce type de disposition fait pourtant débat. Quant à la question de savoir si la politique a un genre, la construction sociale des sexes elle-même est porteuse de politique au féminin et renferme donc le potentiel pour que les femmes puissent effectivement faire la politique autrement.

L'apport de Pascale BOISTARD (2014), mérite d'être mentionné dans cet article. Mais à ce niveau, il est important de signaler que la partie qui attire notre curiosité est sa contribution sur les obstacles persistants à la contribution des femmes en politique. BOISTARD mentionne qu'historiquement, les femmes ont été tenues à l'écart des instances du pouvoir. Même si ce constat de la sous-représentation des femmes en politique est aujourd'hui largement partagé et déploré, la politique reste un univers largement dominé par les hommes, que ce soit au niveau local ou national.

Encore aujourd'hui, les femmes doivent affronter une multitude d’obstacles pour entrer en politique. Il déplore que depuis les années 1990, les études sur ce sujet se sont multipliées. Elles expliquent la sousreprésentation des femmes par différents facteurs :

\section{Des entraves structurelles causées par des pratiques discriminatoires}

$>$ Les barrières socioculturelles : poids des préjugés et perceptions culturelles concernant le rôle des femmes ;

$>$ Le fonctionnement des partis politiques : manque de promotion des candidates, difficulté à obtenir une position éligible ; 
$>$ Le système électoral qui limite le renouvellement du personnel politique.

\section{Des facteurs pesant sur la prise de décision et l’investissement des femmes}

$>$ Un moindre accès à l'éducation et à la formation ;

$>$ Les responsabilités familiales incompatibles avec le temps politique ;

$>$ Le manque de moyens financiers et de réseau de solidarité.

Le rapport du PNUD sur Promouvoir les rôles des femmes pour renforcer les partis politiques apporte d'éléments nouveaux sur les autres. Ce rapport mentionne que les partis politiques sont la clé de la participation politique des femmes, car ce sont eux qui recrutent et choisissent les candidats aux élections et décident de l'agenda politique du pays. Et pourtant, si les femmes sont généralement bien représentées dans les partis au niveau local ou dans des rôles subalternes, elles sont sous-représentées aux postes de pouvoir. Comme elles n’ont pas accès aux réseaux d'influence en place, qu'elles disposent de très peu de ressources, qu'elles ont peu de modèles à suivre ou de mentors et parfois ne bénéficient même pas du soutien de leur famille ou de leur communauté, on peut comprendre que leur participation à la vie politique reste largement inférieure à celle des hommes. La présence des femmes au sein des partis politiques - et la façon dont ceuxci encouragent et cultivent leur participation ou abordent les questions liées à l'égalité des sexes - est un facteur clé de leur émancipation politique. Elle joue aussi un rôle essentiel pour inciter la société toute entière à se préoccuper de l’égalité des sexes. Pour donner de bons résultats, les données de ce rapport précisent que les stratégies encourageant la participation des femmes à la vie politique devraient être liées aux mesures que les partis peuvent prendre aux cours des différentes phases du cycle électoral (stade pré-électoral, électoral et post-électoral) ainsi qu’à l’organisation et au financement des partis eux-mêmes. femmes?

Qu'en est-il des textes qui parlent de la participation politique des

Plusieurs textes tant au plan international que national ont été conçus dans le cadre de la promotion du genre. Ainsi, on distingue:

\section{Au niveau international}

Sous l'égide notamment des Nations unies, la communauté internationale s'est dotée d'un appareil très complet de conventions internationales et de programmes d'action en faveur de l'égalité entre hommes et femmes, qui doivent être retranscrits aux niveaux des Etats et des sous-régions, comme l’Union européenne. 
A partir de 1975, l’ONU lance la Décennie des Nations unies pour la femme (1975-1985). Une série de conférences internationales est mise en œuvre aboutissant à des programmes d'action. En 1995, la Conférence internationale de Pékin sur les femmes définit douze domaines critiques avec des politiques publiques à mettre en œuvre et des objectifs précis à atteindre dans chaque domaine.

Ainsi des « Journées internationales » furent instaurées. L’ONU a proclamé le 8 mars "Journée internationale des femmes", le 25 novembre "Journée internationale pour l'élimination des violences à l'égard des femmes », et le 11 octobre "Journée internationale des filles".

Ensuite, La CEDEF de 1979 fut mise en place. Fruit d'un aboutissement de trente ans de travail de la Commission des Nations unies sur le statut des femmes, la Convention de 1979 sur l'élimination de toutes les formes de discrimination à l'encontre des femmes (CEDEF), constitue l'accord international le plus complet sur les droits humains fondamentaux des femmes.

Elle définit la discrimination à l'égard des femmes comme «toute distinction, exclusion ou restriction fondée sur le sexe qui a pour effet ou pour but de compromettre ou de détruire la reconnaissance, la jouissance ou l'exercice par les femmes, quel que soit leur état matrimonial, sur la base de l'égalité de l'homme et de la femme, des droits humains et des libertés fondamentales dans les domaines politique, économique, social, culturel et civil ou dans tout autre domaine » (Article 1).

Enfin, il faut souligner le Programme d'action de la Conférence de Pékin de 1995. La Conférence mondiale de Pékin sur le statut des femmes tenu en 1995, a marqué une rupture dans la manière de prendre en compte les intérêts des femmes dans l'aide au développement et leur participation aux processus de développement.

Elle a définit des objectifs dans douze domaines: Femmes et pauvreté ; Éducation et formation des femmes ; Femmes et santé ; Violence à l'égard des femmes; Femmes et conflits armés; Femmes et économie ; Femmes et prise de décisions; Mécanismes institutionnels œuvrant à la promotion de la femme; Droits fondamentaux de la femme; Femmes et médias ; Femmes et environnement ; Jeunes filles.

\section{Au niveau national}

$\mathrm{Au}$ Niger, des efforts ont été consentis par les différents gouvernements qui se sont succédé dans le cadre de la promotion des droits de la femme en général et de leur participation politique en particulier de l'indépendance à nos jours.

- Politique Nationale de genre, adoptée en juillet 2008, fortement axée sur les «interrelations entre le genre et le développement», la 
stratégie a pour finalité de contribuer à la réalisation des objectifs d'égalité et d'équité entre les sexes énoncés dans la SDRP. Elle est articulée autour de quatre(4) domaines ;

- Plan décennal de mise en œuvre de la politique nationale de genre 2009-2018;

- Documents stratégiques sectoriels incluant la promotion de l'égalité de genre dans leurs actions et objectifs : (PDES) Plan de Développement Economique et Social 2012-2015 et le PDDE (2003-2013) Programme Décennal de Développement de l'Education.

\section{Loi sur le quota}

La question de la représentativité des femmes dans les instances décisionnelles refait surface après les élections législatives et locales d'octobre et de décembre 2009. Aux dernières législatives, seulement 11 femmes étaient élues sur un total de 113 députés au parlement. En 2004, elles étaient 14 députées pour le même nombre de sièges. Pourtant, selon les statistiques de l'Institut national de la statistique (INS), les femmes représentent plus de 50 pour cent de la population nigérienne estimée à environ 17 millions d'habitants.

Face aux inégalités cruciales qui existent entre les femmes et les hommes dans leur participation à la vie publique, le gouvernement nigérien a approuvé en 1996 une politique nationale de la promotion de la femme.

Elle a abouti en 2000 à l'adoption d'une loi sur le quota qui fixe un seuil minimal de participation de chaque sexe à la gestion des affaires publiques. Aux termes de cette loi, la proportion des candidats élus de l'un ou de l'autre sexe ne doit pas être inférieure à 10 pour cent dans la proclamation des résultats définitifs d'une élection législative ou locale. De même, lors de la nomination des membres du gouvernement et de la promotion aux emplois supérieurs de l'Etat, cette proportion ne doit pas être inférieure à $25 \%$. La loi sur le quota, entrée en vigueur en 2002, a beaucoup favorisé l'émergence politique des femmes pendant les élections générales de 2004. Quatorze femmes étaient élues députées, soit une proportion de 12 pour cent, alors qu'en 1999, sur les 83 députés, il n’y avait qu'une seule femme élue. Aux municipales de 2004, 671 d'entre elles devenaient conseillères sur 3.747 postes à travers les 256 communes du Niger. Elles sont au nombre de 29 sur les 171 députés élus aux dernières élections législatives de 2016 soit $16.96 \%$. 


\section{Résultats}

\section{Participation des femmes au développement politique du Niger}

Il convient de préciser que, conscientes de leur condition de vie, les femmes au Niger n’ont pas gardé les bras croisés pour participer au développement du pays. Longtemps mises de coté dans toutes les entreprises et initiatives nationales, les femmes ont commencé petit à petit à revendiquer leurs droits. On ne peut donc pas parler de participation au développement politique sans pour autant souligner la contribution du genre féminin aux divers secteurs de développement au Niger. Aussi, en démocratie, la participation politique se fait essentiellement au niveau des partis politiques qui sont les structures chargées de participer aux élections pour conquérir et exercer le pouvoir. Analyser donc de la participation politique de la femme au Niger, c'est analyser les différentes activités partisanes qu'elles mènent au sein des partis politiques.

\section{Représentativité des femmes et des hommes dans les instances décisionnelles de partis politiques}

La loi sur le quota (10\%) aux postes électifs et de 25\% aux postes de nomination fait l'objet d'interprétations controversées. Aussi, le mode de désignation des personnalités féminines véritablement représentatives est à beaucoup d'égard discutable. A la méconnaissance des lois ou le refus de les observer, la mise en évidence réelle d'une politique genre, n’est pas la pratique la mieux partagée aussi bien dans les instances de prise de décision des partis politiques qu’à travers le pays de manière générale. Ces résultats rejoignent celles de Sabine (2013) dans ses analyses.

Les stéréotypes, les clichés défavorables sur les capacités des femmes à assumer des responsabilités politiques ainsi que des résistances aux changements intimement liés aux us et coutumes en vigueur dans nombre des régions du pays (si possibles citez les régions qui sont en tête selon les données de cette recherche), justifient à beaucoup d’égard cette situation qui tranche avec le poids démographique des femmes au Niger. En atteste le tableau ci-dessus.

Titre: Les femmes dans les gouvernements de 1990 à 2010

\begin{tabular}{|c|c|c|c|}
\hline $\begin{array}{c}\text { Régime politique } \\
\text { république 1991: 2ème }\end{array}$ & $\begin{array}{c}\text { Total des membres du } \\
\text { gouvernement }\end{array}$ & $\begin{array}{c}\text { Nombre des } \\
\text { femmes }\end{array}$ & $\begin{array}{c}\text { Ratio } \\
\%\end{array}$ \\
\hline $\begin{array}{c}\text { 24 Septembre 1 Juillet } \\
\text { 2 Juillet-15 Avril 1993: Transition } \\
\text { démocratique }\end{array}$ & 25 & 1 & 4 \\
\hline $\begin{array}{c}\text { 16 Avril 1 3 -20 Février 1995 : 3è } \\
\text { république }\end{array}$ & 24 & 2 & 8,3 \\
\hline $\begin{array}{c}\text { 21 Février 1 5 -26 Janvier 1 6: } \\
\text { Cohabitation }\end{array}$ & 28 & 5 & 17,8 \\
\hline $\begin{array}{c}\text { 27 Janvier 1 6-7 Aout 1996: Transition } \\
\text { CSN }\end{array}$ & 16 & 2 & 12,5 \\
\hline \begin{tabular}{c} 
CSN \\
\hline
\end{tabular} & 24 & 4 & 17 \\
\hline
\end{tabular}




\begin{tabular}{|c|c|c|c|} 
8 Aout 1 6-8 Avril 1999 : 4è république & 24 & 3 & 13 \\
\hline $\begin{array}{c}\text { Avril 1999-21 Décembre 1999 : } \\
\text { Transition CRN }\end{array}$ & 20 & 2 & 10 \\
\hline $\begin{array}{c}\text { 5è République: 21 Décembre-2001: 1er } \\
\text { gouvernement }\end{array}$ & 24 & 2 & 13,33 \\
\hline 2001-2004: 2è gouvernement & 28 & 4 & 14,2 \\
\hline 6è république & 30 & 7 & 26 \\
\hline Transition CSRD 2010 & 20 & 5 & 25 \\
\hline 7ème république: 1er gouvernement & 24 & 6 & 25 \\
\hline 2è gouvernement & 24 & 5 & 20 \\
\hline
\end{tabular}

Source : enquête du terrain du 01 au 30 septembre 2016

Au nombre des raisons invoquées qui justifieraient cette situation, les résultats exposent le peu de combativité des femmes pour se faire entendre et prendre conséquemment la place qui leur revient de droit dans la sphère politique. De soixante-cinq partis politiques que compte le pays en 2012, trois seulement sont crées et dirigés par des femmes. De plus, malgré la proportion des femmes parmi la population nigérienne, aucune de ces trois leaders n'occupe actuellement un poste électif. De même aucune d'entre elles n'a franchi la barre de $1 \%$ des suffrages exprimés valables dans toutes les élections présidentielles. Les femmes également sont sous représentées dans les instances de prise de décision des partis politiques et dans les sphères politiques nationales et locales.

Pourtant, le renforcement et la viabilité de la démocratie au Niger passent par la prise en compte de toutes les couches sociales dans la gestion des affaires publiques. Cela justifie l'intérêt pour les décideurs de s'appuyer sur les préoccupations exprimées par les partis politiques eux-mêmes à travers cette forme de diagnostic participatif pour une meilleure efficacité des actions à mener tant au niveau national que local.

\section{Constats}

Titre : répartition du genre selon les partis politiques au Niger

\begin{tabular}{|c|c|c|c|}
\hline Partis politiques & $\begin{array}{c}\text { Représentativité } \\
\text { hommes }\end{array}$ & $\begin{array}{c}\text { Représentativité } \\
\text { femmes }\end{array}$ & total \\
\hline Alfijir & 30 & 30 & 60 \\
\hline Raccin Hadin Kay & 13 & 9 & 22 \\
\hline PNDS & 140 & 23 & 163 \\
\hline MNSD & 188 & 20 & 208 \\
\hline RSD & 53 & 8 & 61 \\
\hline
\end{tabular}

Source : enquête du terrain du 01 au 30 septembre 2016

Sur la base des éléments recueillis en septembre 2016 au sein de l'essentiel des partis politiques, les femmes sont très minoritaires à quelques exceptions près comme au PUND Salama où elles sont au nombre de dix sur les vingt-deux membres que compte le bureau politique national. Un seul 
parti (Alfijir) dont la première responsable est une femme applique la parité avec 30 femmes et 30 hommes dans son bureau politique. Tandis qu'à Raccin Hadin Kay, un autre parti dirigé par une femme, les femmes sont au nombre de neuf (9) sur les 22 membres que compte le bureau politique. Au PNDS (le principal parti au pouvoir), le comité exécutif national compte à son sein 163 membres parmi lesquels on y dénombre que 23 femmes contre 140 hommes. Au MNSD Nassara, sur les 208 membres que compte le bureau politique National, seulement 20 femmes siègent contre 188 hommes. Au RSD Gaskia sur les 61 membres du bureau politique National, 8 seulement sont des femmes contre 53 hommes.

Les femmes sont également sous-représentées dans les instances de prise de décision dans l'écrasante majorité des partis politiques surtout si l'on compare cette présence à leur poids démographique, électoral et social. La politique genre est inexistante au sein de l'essentiel des partis politiques et que, ceux qui l'invoquent ne l'appliquent qu'insuffisamment et /ou selon les circonstances, à l'occasion des consultations électorales aux fins d'engranger des suffrages.

Pourtant, si on part du principe de $10 \%$ de l'un ou l'autre sexe dans les postes électifs, les données au plan national sont encourageantes. Globalement, sur les 4040 élus que compte le Niger, 637 sont des femmes contre 3403 hommes soit 15,76\%.A l'assemblée Nationale, sur un effectif de 171 députés, 29sont des femmes contre 142 hommes soit 16, 96,\%.Au niveau des conseils régionaux sur les 260 conseillers, on y dénombre 36 femmes contre 224 hommes soit 13,84\%.Au niveau des conseils municipaux, sur les 3676 conseillers, 584 sont des femmes contre 3092 hommes soit $15,80 \%$.

S'agissant des conseils des villes, sur les 104 conseillers, 17 sont des femmes contre 87 hommes soit 16,34\% de l'effectif.

On constate également une sous représentation des femmes dans les instances et organes décisionnels des partis politiques. En effet, sur les 1027 membres des bureaux des quatorze partis politiques, les femmes sont au nombre de 196 contre 831 hommes soit 19,08\%.

Ce qui à priori, dépasse les $10 \%$ prévus par la loi sur les quotas, mais n'atteint pas les 25\% consacrés pour les postes nominatifs. Aussi, lorsqu'elles sont membres du bureau, elles sont rarement à des postes de premiers plans dans les partis.

\section{Les femmes dans les bureaux politiques}

L'analyse de la présence des femmes dans les bureaux politiques fait apparaître clairement qu'elles sont nettement en nombre réduit en comparaison au nombre d'hommes composant les bureaux. Les données précisent que même lorsqu'elles sont présentes, elles ne sont presque jamais 
à des postes de direction ou déterminants pour la vie du parti. Ces données nous permettent de voir que seuls trois partis sont présidés par des femmes à savoir: Raccin Hadin Kay (Rassemblement des citoyens pour un Niger nouveau), PNRD Alfijir (Parti Nigérien pour le Renforcement De La Démocratie), MDRPP (Mouvement Démocratique et Révolutionnaire Pour le Pouvoir Peuple).

\section{Tenue des réunions des bureaux politiques}

En ce qui concerne la tenue des réunions tous les partis ont soutenu tenir leurs réunions, les jours non ouvrables en vue de permettre aux travailleurs des différents secteurs de pouvoir y prendre part. Toutefois des réunions extraordinaires peuvent se tenir pendant les jours ouvrables et parfois à des heures tardives lorsque la nécessité et l'urgence l'impose. Or, c'est pendant ces réunions extraordinaires que les plus importantes décisions sont prises. Déjà la tenue des réunions les weekends peinent les femmes du fait que c'est le moment idéal pour les activités social (mariage, baptême...), en plus si c'est tard dans la nuit, elles ne peuvent y prendre part. En général, la femme n’arrive pas à s’investir dans les activités partisanes, de manière constante et conséquente que lorsqu'elle n'a plus le poids de la vie conjugale.

\section{Mécanisme de prise en compte du genre dans les partis politiques}

Selon les résultats de l'entretien exploratoire, L'ensemble des parties soutiennent qu'ils disposent des mécanismes permettant la prise en compte du genre. En outre, les femmes sont régulièrement consultées et impliquées à tous les niveaux de prise de décisions .Cette implication s'opère à travers soit les structures des femmes, soit de manière directe particulièrement sur toutes les questions les concernant.

Parmi les outils utilisés dans ce cadre ,figure la pratique de la parité , le respect de la loi sur le quota, la formation des membres et l' implication à l'élaboration des plans d'action et la mise en place des commissions spéciales, des comité ad 'hoc , et des groupes thématiques.

Cependant quelques partis estiment que le genre est d'abord une question de justice et qu'ils ne disposent pas d'une politique spécifique pour homme et pour femme.

Ainsi des facteurs multiples justifient la position marginale des femmes dans les partis politiques. On y peut citer entre autres :

La crainte de ne pas enregistrer des bons scores électoraux au cas où les femmes seraient au devant de la scène .Cela est surtout observable dans des régions à dominance culturelle patriarcale. Dans la plupart des cas, on ne fait recours aux femmes et aux jeunes qu'en périodes électorales ou pendant des crises politiques pour qu'ils servent des «bras armés " dans 
les combats « des grands » dont souvent ils n'ont pas conscience des vrais enjeux. Cependant, on note de manière croissante une compréhension plus progressive de la dimension genre dans les options et actions des membres des partis politiques au niveau local. Cet espoir d'évolution favorable de la situation, nous inspire quelques suggestions et recommandations.

\section{Contribution aux secteurs de développement}

Les contributions du genre féminin sont multiples et multiformes. Ainsi par secteur, il faut retenir:

Apport au processus démocratique :

L'avènement de la démocratie au Niger, a permis aux femmes de jouer un rôle grâce aux divers postes de responsabilités qu'elles ont occupés. Il s'agit entre autres des nominations à des postes de Directions importantes et ministériels. En outre, beaucoup de femmes ont eu l'honneur d'être élues (Conseillères et/ou Députés).

\section{Apport sur le plan économique, sanitaire, éducatif et syndical}

- La contribution du genre au plan économique n'est pas aussi à négliger, c'est le cas de certaines entreprises dirigées par des femmes (Niger lait, LOLANI, Djamila Pressing, Salon de coiffure...).

- Par rapport au domaine de la santé, on compte plusieurs femmes médecins

- On note aussi sur le plan éducatif, des femmes institutrices, chercheuses...

- En matière syndicale, il faut noter que beaucoup des femmes sont à la tète des organisations de la société civile (CONGAFEN, AFN, ANED, AME...).

\section{Contraintes liées à la participation politique des femmes}

$>$ Les contraintes dans le domaine du genre sont d'ordre budgétaire, institutionnel, juridique et culturel. Elles concernent également l'insuffisance d'information, l'ignorance et la faiblesse des données désagrégées dans le domaine du genre.

$>$ Les discriminations dans le domaine juridique constituent la seconde contrainte à laquelle le genre est confronté (droit coutumier, moderne et religieux) avec une logique différente qui consacre plusieurs discriminations à l'égard de la femme notamment en ce qui concerne leurs droits, les rapports familiaux, les successions, l'accès à la terre, etc.

$>$ La troisième contrainte porte sur l'ignorance des populations (faible taux d'alphabétisation notamment celui des femmes, méconnaissances de leurs droits et ceux de leurs enfants, les opportunités des affaires, 
technologies appropriées nécessaires à la production, la transformation et la conservation des produits,...).

Les pesanteurs socioculturelles qui constituent la quatrième contrainte, font également que les femmes sont victimes de nombreuses violences fondées sur le sexe, notamment les violences conjugales, les mutilations sexuelles, les mariages forcés,...

A titre illustratif on peut citer : l'acceptation pour certains parents d'inscrire leurs enfants à l’école, la fréquentation des formations sanitaires, la formation militaire, le mariage extra communautaire, l'exercice de certains métiers, les habitudes alimentaires pour ne citer que ceux-là. Les pesanteurs les plus connues sont celles qui conduisent à une participation insuffisante à la vie publique. Les contraintes à la progression des femmes en politique sont pour la plupart structurelles : ainsi en est- il de l'idéologie traditionnelle que ni la politique coloniale, ni ensuite les systèmes législatifs nationaux n’ont réussie à supplanter.

\section{Conclusion}

A travers la thématique : «Participation politique des femmes au Niger : Analyse de la contribution du genre féminin dans le développement politique», notre objectif consiste à comprendre et expliquer la contribution des femmes dans le développement politique au Niger. Par ailleurs, il rentre dans un des thèmes de l'actualité politique du Niger tout particulièrement comme le témoignent les ateliers, forums et autres rencontres organisés dans ce cadre. La participation du genre féminin en Afrique en général et au Niger en particulier comme le précise les résultats de cet article est négligeable. Même si la question de la participation politique des femmes est une préoccupation mondiale, au Niger, dans la réalité, cette participation est moindre. S'il est vrai que le droit s'arrache, il n'en demeure pas moins vrai que les combats qu’ont engagés les sociétés civiles, les ONG, partenaires ont eu gain de cause. C'est dire que beaucoup d'acquis ont été obtenus même si par ailleurs les luttes politiques doivent continuer eu égard aux défis à relever dans divers domaines du progrès, de développement.

\section{References:}

1. Abdou Hamani, 2000, « Les femmes et la politique au Niger », édition Démocratie

2. BOISTARD P (2014), femmes et politique, promouvoir l'accès des femmes en politique: la question des quotas, Réseaux femmes parlementaires.

3. Déclaration de la conférence des femmes de la francophonie, Luxembourg, 2000.www.francophonie.org/doc/

Txt- 
référence/decl_luxembourg_2000.pdf. Dictionnaire du féminisme, PUF, 2000.

4. Haut Conseil de la Coopération Internationale, Intégrer le genre dans les actions de coopération et de solidarité internationale, 2006.

5. Foumakoye Nana Aicha, février (2003), Femmes et politiques : expérience du Niger.

6. Manigat S (2013), Participation politique des femmes : qu'est ce qu'on gagne ? Cahier thématique-conditions des femmes haïtiennes.

7. NDI/PNUD, Promouvoir le rôle des femmes pour renforcer les partis politiques.

8. PNUD (2011), Promouvoir le rôle des femmes pour renforcer les partis politiques: guide des bonnes pratiques pour encourager la participation politique des femmes, Graphique services, bureau, inc.

9. Site de Genre en action www.genreenaction.net. 